\title{
Empleo de fichas dirigidas para la mejora en el aprendizaje de las asignaturas Técnicas Instrumentales y Fisicoquímica
}

\author{
Elia María GRUeso Molina
}

Departamento de Química Física

Facultad de Farmacia

Universidad de Sevilla

elia@us.es

ORCID: https://orcid.org/0000-0002-1397-6104

D.O.I.: http://dx.doi.org/10.12795/JDU.2018.i01.16

Pp.: 282-306

\section{Resumen}

Las asignaturas del área de Química Física del Grado en Farmacia, entre las que se encuentran Técnicas Instrumentales (2ㅇ curso, 2ㅇ cuatrimestre) y Fisicoquímica (1er curso, 20 cuatrimestre), ambas de carácter obligatorio, siguen siendo con mucho aquellas que encabezan la lista con mayor porcentaje de suspensos en la titulación, de ahí la necesidad de seguir trabajando en mejorar la asimilación y comprensión de sus contenidos. En este curso académico 2017/2018 se ha propuesto una nueva estrategia basada en el empleo de fichas dirigidas para fomentar el trabajo en grupo de alumnos, y alentar así, su razonamiento deductivo y su capacidad crítica. La innovación empleada fue llevada a cabo en dos ciclos de mejora encadenados, con una duración de 15 
horas cada uno, obteniéndose resultados constatables que denotan la mejora en la asimilación de los contenidos con el consecuente aprendizaje significativo del alumno.

Palabras clave: Fisicoquímica, Técnicas Instrumentales, Grado en Farmacia, Docencia Universitaria, Experimentación Docente Universitaria.

\section{Descripción del contexto}

El primer ciclo de mejora, de 15 horas de duración, se aplica al bloque de prácticas de espectroscopía en la asignatura de Técnicas Instrumentales y participan en él 22 alumnos. Se denota en el grupo la voluntad por aprender y el interés por las fichas. Las clases se imparten en un laboratorio y en 5 sesiones de 3 horas distribuidas semanalmente. Las calificaciones obtenidas pueden suponer un incremento de hasta un punto en la nota final de teoría. Cada alumno realiza en pareja un mínimo de 2 prácticas, y, un máximo de 3 . El segundo ciclo de mejora se aplica al bloque de cinética química perteneciente a las clases teoría de Fisicoquímica, con una duración de 15 horas. En esta actividad participan voluntariamente 22 alumnos de un total de 65 matriculados (47.7\% repetidores). Las clases se distribuyen en 3 horas semanales, y la realización de las fichas, junto con los cuestionarios iniciales y finales al bloque temático, supone un $20 \%$ de la nota final de la asignatura. Adicionalmente, se propuso la realización de casos prácticos pudiendo suponer una mejora en la nota final de la asignatura de hasta 1.0 punto. En ambos casos se denotan fuertes deficiencias en matemáticas y física.

\section{Diseño Previo de los Ciclos de Mejora Encadenados}

En el ciclo de mejora aplicado durante el primer cuatrimestre de este curso, se evidenció que las actividades en forma de casos prácticos daban muy buen resultado y 
fueron muy bien acogidas por los estudiantes. Prueba de ello es la demanda que realizaron de más casos, aunque de mayor simplicidad y claridad en su planteamiento. Basándome en este punto y tratando de solventar la falta de motivación general del alumnado, he propuesto durante este cuatrimestre una adaptación de los casos, que se presentan en estos nuevos ciclos de mejora encadenados en formato de fichas de tareas dirigidas (Finkel, 2008).

\section{Prácticas y teoría implicadas}

El primer ciclo fue aplicado sobre las siguientes prácticas:

1) Determinación de la constante de acidez del indicador Rojo de Metilo (Espectroscopía de absorción UV-visible; duración estimada: 6-9 h)

2) Determinación simultánea de la concentración de cromo y cobalto en una mezcla problema (Espectroscopía de absorción UV-visible; duración estimada: 6h)

3) Determinación de la concentración de quinina en agua tónica (Espectroscopía de fluorescencia; duración estimada: $6 \mathrm{~h}$ )

4) Determinación de sodio y potasio en muestra de suero por espectrometría de absorción atómica (Espectroscopía de absorción atómica o de llama; duración estimada: 3 h)

Hay que destacar que en grupos anteriores he encontrado ciertas deficiencias en el grado de aprendizaje autónomo del alumnado, en la representación gráfica empleando escalas, así como en el grado de comprensión de algunos conceptos teóricos y de ejecución de cálculos numéricos que implican una reflexión profunda sobre los contenidos. No obstante, la actitud del alumno solía ser buena, ya que mostraban interés en la realización de las 
prácticas y se implicaban bastante, por lo que fueron buenos receptores de la innovación propuesta.

Por su parte, el segundo ciclo de mejora consta de los temas de Cinética Formal (8 h), Cinética Molecular (3 horas) y Catálisis (4 horas). En esta asignatura, a diferencia de lo que sucedía en las clases prácticas de Técnicas, se denotó un bajo interés del alumnado por el aprendizaje, siendo muy frecuente el absentismo. Todo ello, probablemente, es debido al fracaso previo registrado en la asignatura de Física Aplicada. Aún así, los alumnos se mostraron bastante receptivos a participar en el trabajo con las fichas y cuestionarios propuestos, aunque, comparativamente, mostraron menos interés que en la asignatura de Técnicas Instrumentales.

\section{Mapa de contenidos y preguntas clave}

En ambos casos, se llevó a cabo una reflexión previa y profunda sobre los contenidos a trabajar, englobándolos en mapas de contenidos y preguntas. Es destacable el trabajo de seleccionar los contenidos organizadores, en asignaturas tan densas y de difícil comprensión, donde todo está relacionado. De hecho, como se ha constatado en múltiples ocasiones, a veces es mejor sacrificar parte de los contenidos en aras de la reflexión del alumno y su implicación (Stephen, 2009). Un ejemplo de ello lo constituye el mapa de la Figura 1, para el primer ciclo de mejora, que engloba las 4 prácticas propuestas. 


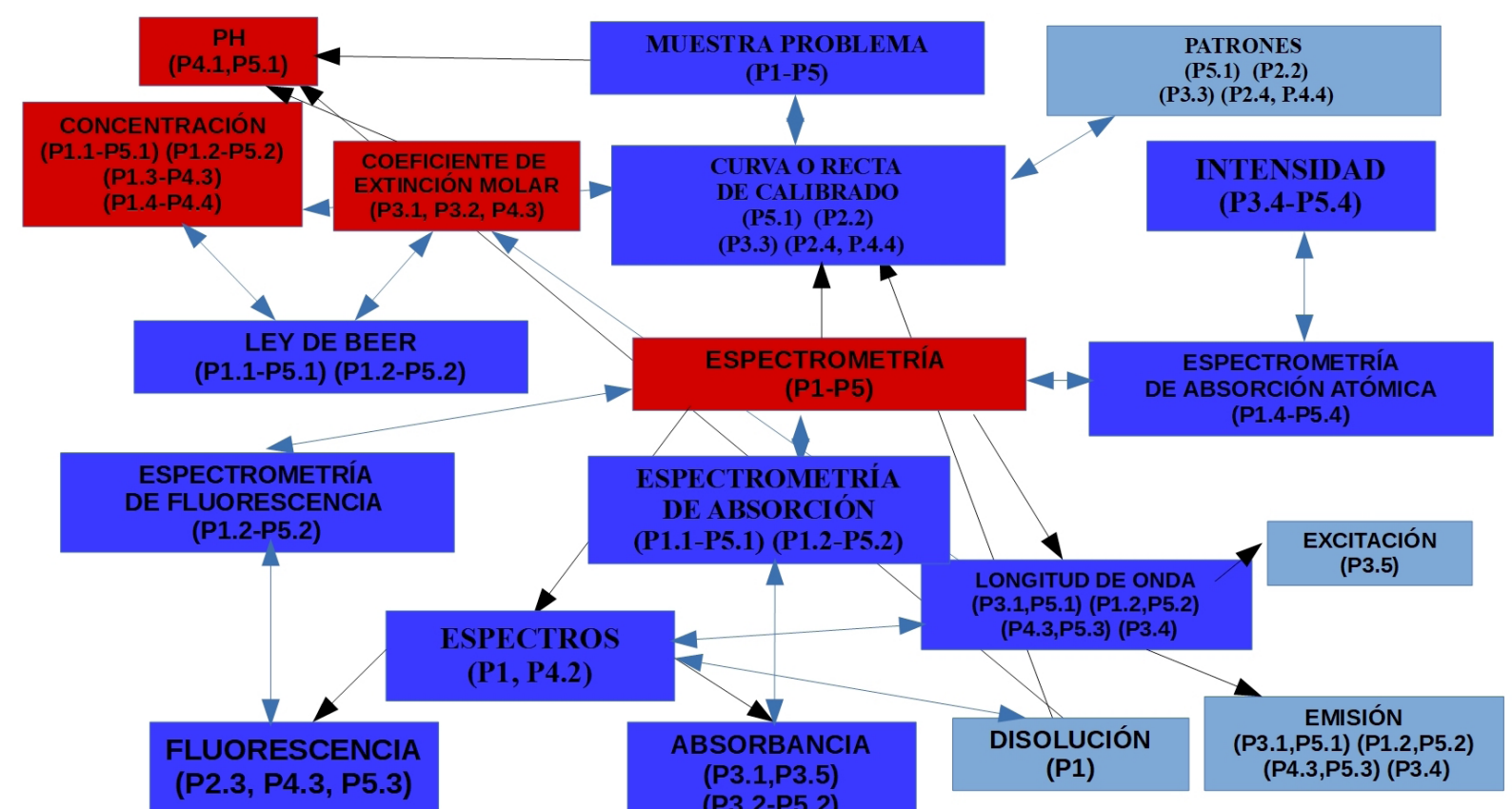

PROCEDIMENTALES GENERALES: Aprender a expresar un resultado de forma correcta con sus unidades y el error asociado; Aprender a trabajar el cambio de unidades; Aprender a construir rectas de calibrado y a trabajar con escalas adecuadas; Aprender a preparar disoluciones por pesada y por dilución; Poder explicar de manera comprensible los fenómenos y procesos que suceden a las muestras patrón y problema; Adquirir destreza en el manejo de programas informáticos para el tratamiento de datos experimentales.

PROCEDIMIENTOS INTELECTUALES:Aprender a distinguir resultados probables de improbables; Fomentar el pensamiento deductivo e inductivo; Adquirir manejo en la preparación de disoluciones y en el manejo del material volumétrico del laboratorio; Interés por el control y manejo de los residuos químicos acumulados en el desarrollo de las prácticas.

ACTITUDINALES GENERALES: Desarrollo de Actitud Crítica y Colaborativa; Desarrollo de iniciativas para resolver problemas en ciencia y de espíritu emprendedor.

Figura 1. Mapa de contenidos empleado en el primer ciclo de mejora correspondiente al bloque de prácticas de espectroscopía de la asignatura de Técnicas Instrumentales

Jornadas de Formación e Innovación Docente del Profesorado | № 1 (2018)

(c) $($ ) $\Theta$ Esta obra se distribuye con la licencia Creative Commons Internacional (CC BY-NC-ND 4.0.) 
En este mapa de contenidos y preguntas se engloban los conceptos que se trabajarán en las diferentes prácticas del bloque de espectroscopía, así como las preguntas asociadas a cada práctica en concreto. La simbología que se emplea (Pj.i) indica el número de la pregunta (j) y la práctica a la que se refiere (i). En el caso de que la/s preguntas se repita/n en las distintas prácticas el símbolo (i) es obviado. La flecha simple indica el sentido normal de la relación entre contenidos, y la flecha doble implica que ambos términos están íntimamente relacionados, pudiéndose llegar de uno a otro en cualquier sentido. En azul oscuro aparecen los conceptos organizadores, en celeste los secundarios, en rojo los contextuales, y en los recuadros inferiores los procedimentales y actitudinales trabajados. En las Tablas 1 y 2 se recogen algunas de las preguntas que se abordaron en los cuestionarios inicial y final en el primer y segundo ciclo de mejora, respectivamente. Nótese que en el primer ciclo las preguntas se recogen en papel a modo de fichas de trabajo en cada práctica.

Jornadas de Formación e Innovación Docente del Profesorado | № 1 (2018) Esta obra se distribuye con la licencia Creative Commons 
Relación de preguntas incluidas en el primer ciclo de mejora a modo de fichas de trabajo en dos de las prácticas trabajadas.

\begin{tabular}{|c|c|}
\hline $\begin{array}{l}\text { Determinación de la Constante de } \\
\text { Acidez del Rojo de Metilo }\end{array}$ & $\begin{array}{c}\text { Determinación de la concentración de } \\
\text { Quinina en Agua Tónica }\end{array}$ \\
\hline $\begin{array}{l}\text { P1. ¿Qué especie/s están presentes } \\
\text { en la disolución problema? ¿Se pue- } \\
\text { den determinar sus concentraciones } \\
\text { de forma simultánea? En caso afir- } \\
\text { mativo indique cómo lo haría. }\end{array}$ & $\begin{array}{l}\text { P1. ¿Qué especie/s están presentes en } \\
\text { la disolución problema? ¿Se puede de- } \\
\text { terminar la quinina en presencia del } \\
\text { resto de componentes de la disolución? }\end{array}$ \\
\hline $\begin{array}{l}\text { P2. ¿Qué técnica instrumental me } \\
\text { permitiría determinar la concentra- } \\
\text { ción de las formas ácida y básica? ¿Es } \\
\text { necesario realizar un calibrado pre- } \\
\text { vio? ¿Vale mi curva de calibrado para } \\
\text { otra pareja del grupo? }\end{array}$ & $\begin{array}{l}\text { P2. ¿Qué particularidad tienen las sus- } \\
\text { tancias que emiten fluorescencia en re- } \\
\text { lación con su estructura? }\end{array}$ \\
\hline $\begin{array}{l}\text { P3. ¿Qué ley relaciona absorbancia } \\
\text { con concentración? Indique la varia- } \\
\text { ble principal de la que depende el } \\
\text { coeficiente de absortividad molar. }\end{array}$ & $\begin{array}{l}\text { P3. ¿Qué técnica instrumental me per- } \\
\text { mitiría determinar la concentración de } \\
\text { quinina? ¿Es necesario realizar un ca- } \\
\text { librado previo? ¿Vale mi calibrado para } \\
\text { otra pareja del grupo? }\end{array}$ \\
\hline $\begin{array}{l}\text { P4. A partir de la expresión de la } \\
\text { constante de equilibrio para el rojo } \\
\text { de metilo determine la relación en- } \\
\text { tre el pH y las concentraciones de la } \\
\text { forma ácida y básica. }\end{array}$ & $\begin{array}{l}\text { P4. ¿Cómo se relaciona la fluorescencia } \\
\text { con la concentración a una longitud de } \\
\text { onda dada? ¿Se cumple esta relación } \\
\text { a cualquier concentración de muestra? } \\
\text { Indique la variable principal de la que } \\
\text { depende el coeficiente de absortividad } \\
\text { molar. }\end{array}$ \\
\hline $\begin{array}{l}\text { P5. Dibuje un esquema de cómo es- } \\
\text { pera que varíe la absorbancia de } \\
\text { la especie básica y ácida del rojo } \\
\text { de metilo en función del pH de } \\
\text { la disolución. ¿Existe algún punto } \\
\text { característico? }\end{array}$ & $\begin{array}{l}\text { P5. En espectroscopía de fluorescen- } \\
\text { cia la especie fluorescente pasa a un } \\
\text { estado excitado al ser irradiada con } \\
\text { la longitud de onda de excitación y } \\
\text { después decae al estado fundamen- } \\
\text { tal previa desactivación desde un ni- } \\
\text { vel energético inferior. En base a ello } \\
\text { ¿Cómo será la longitud de onda del } \\
\text { máximo de emisión en el espectro en } \\
\text { relación con la longitud de onda de } \\
\text { excitación?i }\end{array}$ \\
\hline
\end{tabular}


Por otra parte, en el segundo ciclo de mejora, además de las preguntas del cuestionario inicial y final recogidas en la Tabla 2, se propusieron otras fichas de trabajo (véase Figura 2) con el objetivo de reforzar y trabajar cada una de las cuestiones clave, planteadas en el cuestionario. Esto es, en base a problemas concretos que implicaban una reflexión profunda sobre los contenidos. Estas fichas, que incluían indicaciones o pistas del profesor para ayudar a abordar y discutir la problemática, se repartieron en clase en formato papel. 
Tabla 2. Relación de preguntas, incluidas en los cuestionarios inicial y final, correspondientes al segundo ciclo de mejora aplicado al bloque de Cinética Química.

\begin{tabular}{|c|c|}
\hline \multirow[t]{2}{*}{ P. № } & Ciclo de Mejora 2 \\
\hline & Cinética Química \\
\hline 1 & $\begin{array}{l}\text { ¿Qué opinión te merecen las siguientes afirmaciones? ¿Estás de } \\
\text { acuerdo con ellas? ¿Por qué? ¿Crees que puedes hacer una for- } \\
\text { mulación mejor? Desarróllala y justificala. } \\
\text { a) Para la reacción genérica global A } \rightarrow \text { P se encuentra que la re- } \\
\text { presentación gráfica del ln[A] vs. t (tiempo), presenta una de- } \\
\text { pendencia lineal, por tanto, puede afirmarse con total certeza } \\
\text { que el mecanismo es sencillo y ocurre en un solo paso. } \\
\text { b) Para la reacción genérica global que transcurre en una sola } \\
\text { etapa: } A \rightarrow \text { P, se encuentra que la representación gráfica del } \\
\text { In[A] vs. t (tiempo) presenta una dependencia lineal, por tanto, } \\
\text { puede afirmarse que la cinética es de primer orden respecto del } \\
\text { reactivo A. } \\
\text { c) Dada la reacción global: } 1 \mathrm{~A}+2 \mathrm{~B} \rightarrow \text { C debe cumplirse que } \mathrm{v}_{\mathrm{B}}= \\
1 / 2 \mathrm{v}_{\mathrm{A}} \text {. } \\
\text { d) Si al determinar la velocidad de reacción respecto de un pro- } \\
\text { ducto y de un reactivo en la misma reacción estas resultan ser } \\
\text { distintas, entonces puede afirmarse que el mecanismo de reac- } \\
\text { ción es complejo. }\end{array}$ \\
\hline 2 & $\begin{array}{l}\text { Seleccione con una cruz la respuesta que considere más apro- } \\
\text { piada y razónela. ¿Crees que puedes desarrollar una respuesta } \\
\text { mejor? Si es así, reformula la respuesta y justifica el/los cam- } \\
\text { bio/s realizados. } \\
\text { - El método de las velocidades iniciales es conveniente cuando } \\
\text { la reacción objeto de estudio presenta una cinética muy lenta. } \\
\text { Dada la reacción genérica: } 2 A+3 B \rightarrow \text { C, aplicar el método de ais- } \\
\text { lamiento de Ostwald para determinar el orden parcial respecto } \\
\text { del reactivo A supone.... } \\
\text { a) realizar experimentos cinéticos a concentraciones muy eleva- } \\
\text { das de A (en exceso de A) y determinar la velocidad de desapa- } \\
\text { rición de B a distintas concentraciones. } \\
\text { b) realizar experimentos cinéticos a concentraciones muy eleva- } \\
\text { das de B (en exceso de B) y determinar la velocidad de desapa- } \\
\text { rición de A a distintas concentraciones. } \\
\text { c) trabajar en exceso de ambos reactivos A y B. } \\
\text { - La presencia de un catalizador en el mecanismo de reacción: } \\
\text { a) Disminuye la energía de activación y aumenta el factor de } \\
\text { frecuencia. } \\
\text { b) Aumenta la energía de activación y el factor de frecuencia. } \\
\text { c) Disminuye la energía de activación y no modifica el equilibrio. }\end{array}$ \\
\hline
\end{tabular}


3 Para el siguiente mecanismo indique cómo obtendría la ecuación de velocidad, siendo la reacción global: $2 A \rightarrow 2 B+C$

$$
\begin{gathered}
\mathbf{A} \stackrel{\mathbf{k}_{1}}{\underset{\mathbf{k}_{-1}}{\longrightarrow}} \mathbf{B}+\mathbf{M}^{*} \\
\mathbf{A}+\mathbf{M}^{*} \stackrel{\mathbf{k}_{2}}{\longrightarrow} \mathbf{B}+\mathbf{C}
\end{gathered}
$$

¿Qué papel cree que juega la especia $M^{*}$ en el mecanismo? ¿Cuál cree que sería el orden global si la etapa 2 es una etapa muy rápida en comparación con la primera?

Luego se trabajaron las fichas en clase en grupos reducidos de alumnos (un máximo de 3) y se entregaron al finalizar para su evaluación (véase Figura 2). En todo momento existió retroalimentación con el profesor para resolver las dudas. Se propusieron 6 fichas, las fichas 1-4 en el tema de Cinética formal, más amplio y fundamental, la ficha 5 para el tema de Cinética Molecular y la ficha 6 en el tema de Catálisis.

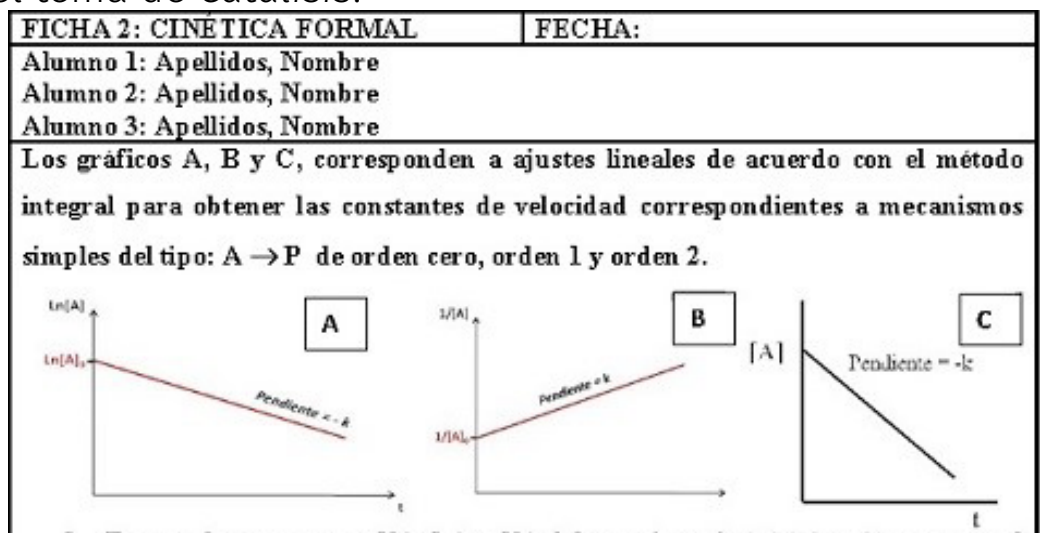

1. Retome las preguntas $1 \mathrm{~b}$ ), $1 \mathrm{~g}$ ) y lh) del cuestionario inicial, $\mathbf{y}$ discuta con el compañero cuál cree que será el orden de reacción que predice cada gráfico.

2. ¿Cómo obtendría la concentración inicial de $\mathrm{A}$ a partir de la representación gráfica? ¿Y la constante de velocidad?

3. Si fuese de orden uno la cinética, ¿cómo sería la expresión de la ecuación de velocidad?. Integre dicha expresión igualándola a la velocidad de desaparición de A.

4. Haga la misma operación sup oniendo orden 0 y 2 .

5. Vuelva a discutir con el compañero la pregunta 1 . ¿Cuál es la conclusión final?

Figura 2. Imagen de la Ficha 2 trabajada con los alumnos de las clases de teoría de Fisicoquímica durante el segundo ciclo de mejora aplicado al bloque de Cinética. 


\section{Modelo Metodológico}

El modelo metodológico empleado en el primer ciclo parte de la base del aplicado en el primer cuatrimestre durante las clases de teoría de Física Aplicada, siendo adaptado en algunos aspectos a las prácticas de Técnicas Instrumentales. Básicamente, se distinguen en él 4 etapas o momentos diferentes en la práctica docente (véase Figura 3, ciclo de mejora 1):

- Etapa 1 (color azul): Se planteó el problema asociado a la práctica, se realizó una lectura razonada del guión y se evaluaron las ideas previas del alumno mediante el cuestionario inicial. La Tabla 1 muestra la coincidencia de algunas preguntas y la especificidad de otras.

- Etapa 2 (color rojo): Se describieron y debatieron los objetivos de cada práctica en grupo, se realizó una breve explicación teórica sobre los contenidos a trabajar. La explicación teórica debe ser entendida como un contraste de ideas, entre las ideas previas del estudiante (trabajadas en el cuestionario inicial) y del profesor.

- Etapa 3 (color verde): El alumno ejecutó la práctica, empleó el equipo de medida necesario (análisis instrumental), realizó la recta o curva de calibrado para resolver el problema y obtuvo el dato final. Con todo ello el alumno confirmó o no las hipótesis planteadas en la etapa 2, comprobando cuales de ellas resultaron más exitosas. Adicionalmente, el profesor planteó cuestiones adicionales para mejorar la comprensión de la práctica.

- Etapa 4 (color amarillo): El alumno resolvió de nuevo el cuestionario inicial que adjuntó al informe final de la práctica.

En cambio, el modelo empleado en el segundo ciclo (véase figura 3) se aplicó a las clases teóricas de 
Fisicoquímica. Este modelo, basado en el anterior, también incluía las fichas de trabajo como novedad, debido a su éxito en el ciclo anterior. Algunas de las modificaciones que se introdujeron fueron: (i) se empleó el mapa de contenidos como base para la descripción de los contenidos; (ii) se eliminó del cuestionario inicial el acontecimiento histórico, y se sustituyó por un problema de índole farmacéutica relacionado con el tema y de aplicación práctica actual; (iii) en una segunda fase, de trabajo de los contenidos, se sustituyeron los casos prácticos planteados por el profesor en pizarra, y asociados a cada pregunta clave, por actividades dirigidas con fichas que se trabajaron en grupos de alumnos (véase Figura 2); (iv) la resolución del problema clave planteado al inicio de la lección se comenzó en clase y se terminó en casa, proponiéndose como trabajo; (v) en las conclusiones generales se hizo alusión de nuevo al mapa de contenidos incluyendo las preguntas clave trabajadas. En dicho modelo metodológico se distinguen 3 etapas o momentos diferentes:

- Etapa 1 (color azul): Se plantearon los problemas clave asociados al tema, se describieron los contenidos en base al mapa y se realizó el cuestionario inicial.

- Etapa 2 (color rojo): Se trabajaron los contenidos mediante fichas. La explicación teórica debe ser entendida como un contraste de ideas profesor-alumno.

- Etapa 3 (color verde): El alumno empleó los conocimientos adquiridos en el tema para la resolución del problema clave planteado al inicio, confirmando o no las hipótesis planteadas en la etapa 2. Finalmente, el alumno resolvió de nuevo el cuestionario inicial y se resumieron las conclusiones más relevantes empleando el mapa de contenidos. 


\section{Secuencia de Actividades}

Por su parte, en la Figura 4 se detalla la secuencia de actividades programada para trabajar la actividad 3 y 1, co- $^{-}$ rrespondientes al primer y segundo ciclo de mejora, respectivamente. Como puede verse a partir de la figura, las actividades que se propusieron se basaron en el trabajo con problemas o cuestiones de indole reflexiva planteadas por el profesor.

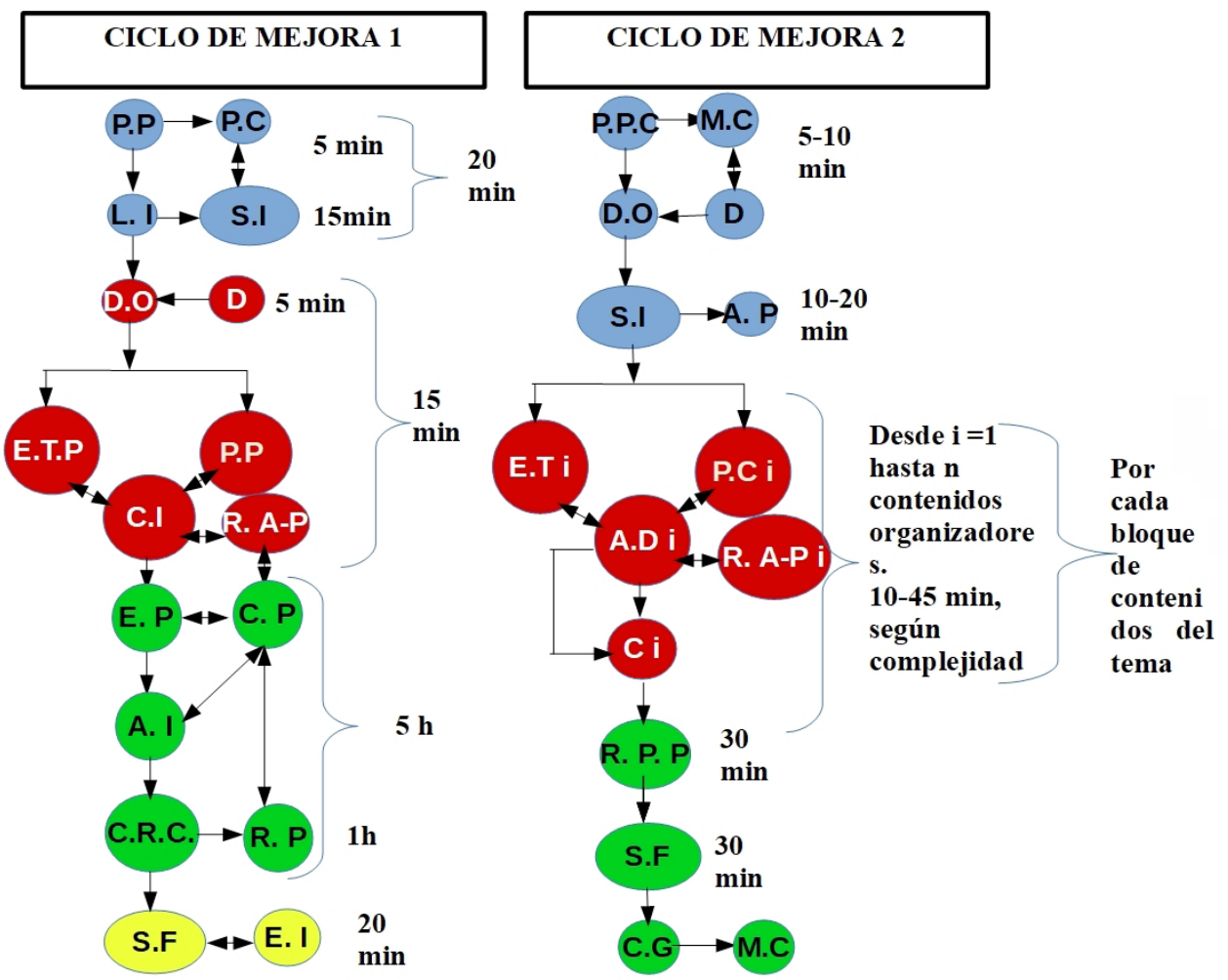

Leyenda de Ia figura: S. I: Sondeo Inicial; S. F: Sondeo Final; L. I: Lectura inicial; P. C: Pregunta clave; P.P: Planteamiento problema asociado a la práctica; D: Debate; D. O: Descripción de Objetivos; E. T. i: Explicación Teórica de la práctica.; R. A-P: Retroalimentación alumno-profesor; C.I: Contraste de ideas; E. P: Ejecución de la práctica; C.P: Cuestiones profesor; A.I: Análisis Instrumental; C. R.. C: Construcción recta o curva de calibrado; R. P: Resolución Práctica; S. F: Sondeo final; E. I: Elaboración de Informes; P.P.C: Planteamiento Problema Clave; R.P.P: Resolución Problema Clavel; A.P: Aplicación práctica; P. C: Pregunta clave i; E. T. i: Explicación Teórica correspondiente al concepto organizador i.; P.C.i: Pregunta clave correspondiente al concepto organizador i.; A.D.i: Actividad dirigida al trabajo de la pregunta clave i en grupos de alumnos; C.i: Conclusiones concepto organizador i.; C.G: Conclusion es generales; M. C: mapa de contenidos.

Figura 3. Esquema del modelo metodológico empleado en los ciclos de mejora.

Jornadas de Formación e Innovación Docente del Profesorado | № 1 (2018)

(c) (i) (2) $\Theta$ Esta obra se distribuye con la licencia Creative Commons Reconocimiento-NoComercial-SinObraDerivada $\quad 4.0$ Internacional (CC BY-NC-ND 4.0.) 


\begin{tabular}{|c|c|}
\hline $\begin{array}{l}\text { ACIVIDAD } \\
\text { N } \% \text { TIEMPO } \\
\text { FSTIMADO }\end{array}$ & CICIOO DE MELOILA 1 \\
\hline $\begin{array}{l}\text { A CTIVID AD } \\
\text { 3. El ahumno } \\
\text { ejecuta la } \\
\text { practica } \\
\text { (de } 1 \text { a } 3 \text { h) }\end{array}$ & 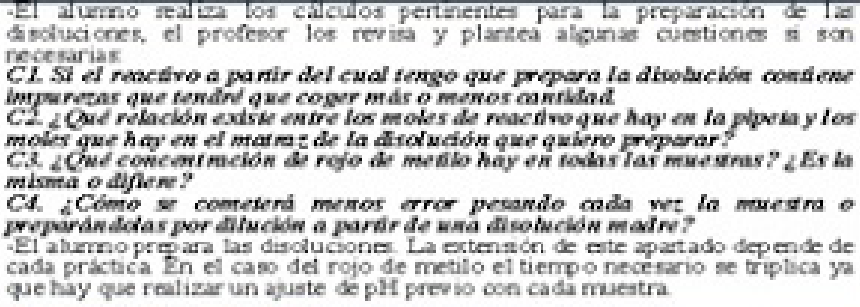 \\
\hline \multirow{4}{*}{$\begin{array}{l}\text { A CTIVIDAD } \\
\text { 1: Problema } \\
\text { clavey } \\
\text { cuestionarbo } \\
\text { inicial } \\
\text { ( } 30 \mathrm{~min})\end{array}$} & CICIO DE MEIORA 2 \\
\hline & 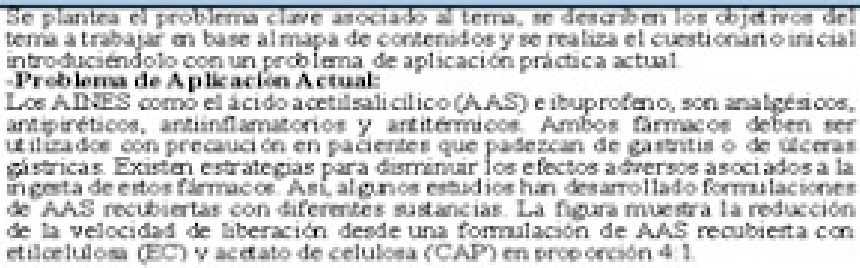 \\
\hline & 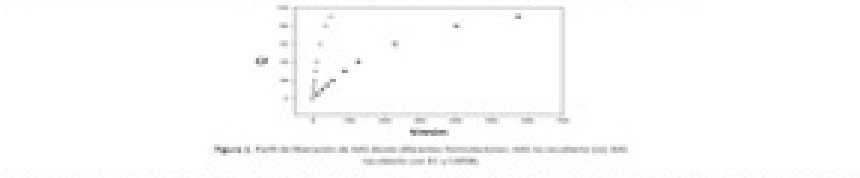 \\
\hline & 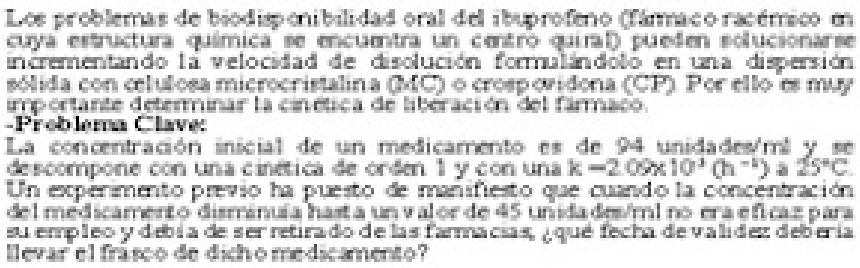 \\
\hline
\end{tabular}

Figura 4. Ejemplo de secuencia de actividades programada en los ciclos de mejora.

\section{Aplicación de los Ciclos de Mejora Encadenados}

Las hojas de actividades empleadas han demostrado ser muy útiles tanto para fomentar la colaboración del alumnado, como para la reflexión crítica sobre los contenidos. Además, se ha logrado simplificar con ellas la tarea de aprendizaje, aportando pistas al alumno para la resolución de los problemas y casos tanto por la retroalimentación alumno-profesor como por la conducción de la reflexión

Jornadas de Formación e Innovación Docente del Profesorado | № 1 (2018)

(c) Esta obra se distribuye con la licencia Creative Commons Reconocimiento-NoComercial-SinObraDerivada $\quad 4.0$ Internacional (CC BY-NC-ND 4.0.) 
del alumno en forma de preguntas que dirigen sus pasos en el razonamiento (Finkel, 2008). Adicionalmente, hay que destacar como novedad el trabajo con fichas tanto en las clases prácticas (primer ciclo de mejora) como en las clases de teoría (segundo ciclo), encontrándose en ambos casos resultados muy gratificantes y prometedores.

A continuación, se detallan algunos aspectos sucedidos en las clases al aplicar la secuencia de actividades programada en el primer ciclo de mejora.

Durante la actividad 1, el alumno leyó el guión de prácticas y contestó a las preguntas propuestas en la ficha de actividades. En el transcurso de esta actividad se observó un mayor interés por la lectura comprensiva del guión de prácticas con respecto a otros grupos donde no se realiza la innovación. Probablemente, esto es debido a que saben que después se trabajará en la puesta en común argumentada de las respuestas e hipótesis dadas a las preguntas.

Posteriormente, en la actividad 2, se realizó la puesta en común de las respuestas a las preguntas del cuestionario, empleando la pizarra como ayuda para las conclusiones y dudas que surgieron. Llama la atención también el hecho de que los alumnos mostraron una actitud más participativa que en la mera explicación práctica con pizarra realizada en otros grupos anteriores. La pregunta 1 de cada práctica se entendió bien y la respondieron bien casi todos los alumnos. En la pregunta 2 tenían el concepto erróneo de que el calibrado realizado vale para todos los alumnos y debe ser el mismo para todas las parejas, de ahí se entiende el interés que mostraron por tienen de obtener resultados idénticos entre sí. Este concepto de calibrado debe trabajarse en base a la experimentación en la actividad 3 para quedar completamente asimilado. La tercera pregunta se trabajó en grupos y se entendió en general bastante bien. Las dos últimas cuestiones más complejas se dejaron abiertas en la explicación y los alumnos comprobaron la validez de sus hipótesis o no durante la realización del experimento. 
Durante la actividad 3, la pregunta: "Si el reactivo a partir del cual tengo que prepara la disolución contiene impurezas que tendré que coger más o menos cantidad" se planteó a los alumnos que tenían problemas al calcular como influye la riqueza de los reactivos en los cálculos de la pesada, y en todos les casos sirvió para resolver sus dudas. La pregunta: “¿Qué concentración de rojo de metilo hay en todas las muestras? ¿Es la misma o difiere?" se realizó a los alumnos cuando dudaron sobre los reactivos que forman parte de las disoluciones-patrón en la práctica del rojo de metilo, lo cual ayudó a responder o aclarar la pregunta 4 del cuestionario (véase Tabla 1). La pregunta: “QQué es mejor medir de la muestra más diluida a la más concentrada o viceversa?, ¿por qué?", se realizó en las prácticas de fluorimetría de la quinina, de llama y Cromo/Cobalto; y la cuestión equivalente que se formuló en la práctica del rojo de metilo: "Si quiero medir la forma básica, ¿cómo procederé de la muestra de mayor $\mathrm{pH}$ a la de menos $\mathrm{pH}$, o viceversa?, ¿por qué?, ¿y si quiero seguir ahora la forma ácida?", se hizo cuando el alumno iba a medir empleando la técnica instrumental correspondiente, para aclarar cuál es la forma más adecuada de trabajar cometiendo menor error en la obtención de los resultados experimentales. Además, sirvieron para aclarar que cuando existe solapamiento de señales es necesario controlar con mayor precisión las condiciones experimentales para lograr una mayor reproducibilidad en las medidas; al tiempo que se profundiza sobre la estrecha relación existente entre señal-concentración, señal-ruido y solapamiento de bandas. Por último, preguntas como: “¿Debe pasar la gráfica por el cero? ¿A qué se debe que la recta de mejor ajuste no pase por el cero?" o "¿Qué escala has elegido para construir la gráfica? ¿Cómo dibujas los puntos y con qué precisión?" sirvieron para que el alumno comprobara la bondad de su representación gráfica y la escala propuesta, cuestionando la validez de la ecuación del ajuste empleada para obtener el resultado final de la medida. En general surgieron dudas acerca de cuál era la escala más idónea y de la relación que existe entre la señal medida y la concentración. 
Finalmente, en la actividad 4, se denotó cierta dificultad para comprender que la concentración de muestra a determinar no es directamente la que miden, sino que depende de la dilución realizada, y que, generalmente, es necesario realizar dichas diluciones para conseguir respuestas lineales señal-concentración. Para facilitar la comprensión de este concepto y la respuesta a la última pregunta de los cuestionarios se guió el razonamiento del alumno con algunas cuestiones como: “¿Has tenido en cuenta la dilución realizada en la preparación de la muestra problema?". Mayoritariamente, y con ayuda de algunas dudas resueltas en la pizarra, el alumno fue capaz de resolver definitivamente la práctica. Es muy destacable que, en general, en el cuestionario final todos los alumnos resuelvan adecuadamente las preguntas de la 1 a la 4; e incluso la pregunta 5 , siendo más compleja.

A continuación, se detallan algunos de los aspectos sucedidos durante la impartición del segundo ciclo en las clases de teoría de Fisicoquímica, destacando sobretodo, lo ocurrido en las actividades donde se proponían fuertes modificaciones en el modelo metodológico: problema de aplicación práctica en el cuestionario inicial y las fichas y conclusiones finales en base al mapa de contenidos. En la actividad 1, se denotó como el problema de aplicación despertó el interés en el alumno por comprender las aplicaciones prácticas que tiene la asignatura en su vida profesional. Una prueba fehaciente de ello fue el aumento, en un $75 \%$, de los casos prácticos presentados por los alumnos frente a lo registrado en Física Aplicada. Durante la realización de las fichas de tareas se destaca en general la necesidad continua del alumnado porque el profesor valide la solidez de sus hipótesis y el contraste de ideas. Determinados grupos de trabajo funcionaron mejor que otros, pero al final, con mayor o menor esfuerzo llegaron a las mismas conclusiones. En general, los alumnos se implicaron bastante en esta actividad, y expresaron que les ayuda a mejorar su grado de aprendizaje. El único problema que encontré fue la falta de tiempo del que se disponía en clase para su realización. De hecho, las fichas, 
que en un principio estaban diseñadas para comenzarlas y finalizarlas en clase, tuvieron que ser, en su mayoría, finalizadas en casa. La ficha 1, por el mismo motivo, se realizó directamente en la pizarra con la participación de todos los alumnos.

A continuación, se detallan algunos de los puntos que fueron más difíciles de comprender y asimilar por los alumnos en cada una de las fichas trabajadas:

En la ficha 1, se observó la dificultad que tienen los alumnos para distinguir entre reacción cinética global y mecanismo de reacción, al final entendieron que varios mecanismos podían ser compatibles con una misma reacción global.

En la ficha 2, se detectaron dificultades en la integración de ecuaciones cinéticas y en establecer la relación entre las representaciones lineales a realizar dependiendo del orden y la ecuación deducida. La ficha estuvo bien planteada y resolvió las dudas.

En el caso de la ficha 3, se encontraron dificultades en establecer la relación entre las unidades de la constante de velocidad y el orden de reacción. Se propone para el futuro realizar un análisis dimensional de la ecuación de velocidad para aclarar la cuestión.

Por su parte la ficha 4, tuvo gran relevancia en cuanto a su complejidad, a la resolución de dudas y al aprendizaje logrado por el alumno, siendo este similar al logrado en la ficha 2. En particular se detectaron dificultades en el alumno a la hora de relacionar las etapas elementales de los mecanismos cinéticos y la expresión de la velocidad de formación o eliminación del intermedio reactivo $X$. Los alumnos se preguntaron ¿qué especies tienen el papel de reactivos en cada etapa elemental?, ¿cómo influye la estequiometría en la concentración y cuando hay que sumar o restar términos de velocidad empleando la hipótesis del estado estacionario?. Concluyo que por el nivel de dificultad de estos conceptos es necesario incluir una ficha adicional en cursos posteriores. 
En la ficha 5 se detectó un error de concepto general en la comprensión de la ecuación de Arrhenius. En concreto, los alumnos suponían la dependencia con T de todos los parámetros de la ecuación. Al final la duda se aclaró. Destaca positivamente el grado de comprensión adquirido sobre las teorías de colisiones y complejo activado.

Finalmente, en la ficha 6 , se aclaró un error de concepto relacionado con la dependencia constante de Michaelis-concentración de enzima. Con retroalimentación alumno-profesor, y recurriendo a la ecuación de van't Hoff, se determinó que la constante es invariable cuando la temperatura no varía, aclarándose la cuestión.

Por último, señalar que la enumeración de las conclusiones del tema en base al mapa de contenidos sobrepasa con mucho la capacidad del alumno de primer curso, y es un tema complejo que debe ser abordado con una estrategia alternativa más sencilla.

La evaluación del aprendizaje del alumno se llevó a cabo, en el primer ciclo, comparando las contestaciones de los alumnos a los cuestionarios, con formato ficha, que fueron entregados al inicio de las prácticas y a la finalización de las mismas. En cambio, en el segundo ciclo de mejora, la evaluación se realizó en base a las preguntas del cuestionario inicial y final, que fueron trabajadas mediante las fichas dirigidas en clase. Esto es, debido a que en clases de teoría se disponía de mayor flexibilidad para la combinación de ambas estrategias en la secuencia de actividades. A modo de ejemplo, se detallan los resultados distribuidos en modelos de pensamiento en la Tabla 3 , para las prácticas de rojo de metilo (ciclo de mejora 1), cuyas preguntas se recogen en la Tabla 1; así como los resultados correspondientes a las preguntas recogidas en la Tabla 2 durante el segundo ciclo de mejora. Los modelos de clasifican de la A (más complejo) a la E (más sencillo y alejado de la realidad física), incluyendo los porcentajes de alumnos en cada nivel. Esto es, tratando de simular los niveles de aprendizaje como peldaños de una escalera, de mayor o menor complejidad, que hay que superar para alcanzar el siguiente nivel. Nótese, que no todos los niveles son detectados en el cuestionario inicial, descubriéndose 
nuevos modelos de pensamiento al finalizar el ciclo. La comparativa de alumnos en uno y otro nivel antes y después de la práctica en el aula denota el grado de avance de la clase y el éxito de los ciclos de mejora.

A partir de los datos de la Tabla 3 cabe señalar que parece que el éxito en el aprendizaje es comparativamente mayor en el primer ciclo de mejora; pues hay una suma mayor en el porcentaje de alumnos distribuidos entre los modelos $A$ y $B$ en el cuestionario final. Esto último, puede deberse a 2 motivos: (i) los alumnos disponen del guión de prácticas que les sirve de ayuda en su razonamiento; (ii) parece que se logra un aprendizaje más significativo si las hipótesis que se plantean se contrastan con procesos de experimentación. A pesar de ello, los resultados en el ciclo de teoría, tras la aplicación de las fichas dirigidas, son también bastante positivos, encontrándose como mínimo, un 81.2 \% de los alumnos en el cuestionario final, distribuidos entre los niveles $A$ y $B$.

Jornadas de Formación e Innovación Docente del Profesorado | № 1 (2018) Esta obra se distribuye con la licencia Creative Commons 
Tabla 3.

Porcentaje de alumnos en los diferentes modelos de pensamiento detectados en algunas de las preguntas trabajadas en los cuestionarios inicial y final.

\begin{tabular}{|c|c|c|c|c|c|c|c|c|}
\hline \multirow{2}{*}{$\begin{array}{l}\text { Pregunta } \\
\text { no/Ciclo } \\
\text { de Mejora }\end{array}$} & \multicolumn{4}{|c|}{ Modelos Iniciales } & \multicolumn{4}{|c|}{ Modelos Finales } \\
\hline & $\% A$ & $\%$ B & $\% \mathrm{C}$ & $\% D$ & $\% A$ & $\%$ B & $\% \mathrm{C}$ & \%D \\
\hline $\begin{array}{l}\text { P1. R.M. } \\
\text { (1 }{ }^{\text {er Ciclo) }}\end{array}$ & 0.0 & 77.3 & 22.7 & $x$ & 72.7 & 27.3 & 0.0 & $x$ \\
\hline $\begin{array}{l}\text { P2. R. M. } \\
\left(1^{\text {er }} \text { Ciclo }\right)\end{array}$ & 0.0 & 81.8 & 18.2 & $x$ & 90.9 & 9.1 & 0.0 & $x$ \\
\hline $\begin{array}{l}\text { P4. Quinina } \\
\text { (1 }{ }^{\text {erCiclo })}\end{array}$ & 0.0 & 31.9 & 13.6 & 54.5 & 85.7 & 14.3 & 0.0 & 0.0 \\
\hline $\begin{array}{l}\text { P5. Quinina } \\
\text { (1 }{ }^{\text {er }} \text { Ciclo) }\end{array}$ & 0.0 & 50.0 & 37.5 & 12.5 & 100 & 0.0 & 0.0 & 0.0 \\
\hline $\begin{array}{l}\text { P1A (20 } \\
\text { Ciclo) }\end{array}$ & 0.0 & 18.8 & 25.0 & 56.3 & 68.8 & 31.2 & 0.0 & 0.0 \\
\hline $\begin{array}{l}\text { P1B (20 } \\
\text { Ciclo) }\end{array}$ & 0.0 & 35.3 & 29.4 & 35.3 & 82.4 & 17.6 & 0.0 & 0.0 \\
\hline $\begin{array}{c}\text { P1C } \\
\text { (2o-Ciclo) }\end{array}$ & 0.0 & 18.8 & 75.0 & 6.2 & 56.3 & 25.0 & 18.8 & 0.0 \\
\hline $\begin{array}{l}\text { P1D (20 } \\
\text { Ciclo) }\end{array}$ & 5.6 & 33.3 & 33.3 & 27.8 & 72.2 & 27.8 & 0.0 & 0.0 \\
\hline $\begin{array}{c}\text { P2A (20 } \\
\text { Ciclo) }\end{array}$ & 11.8 & 29.4 & 58.8 & $x$ & 82.4 & 11.8 & 5.8 & $x$ \\
\hline $\begin{array}{l}\text { P2B (20 } \\
\text { Ciclo) }\end{array}$ & 23.5 & 5.9 & 11.8 & 58.8 & 88.2 & 5.9 & 0.0 & 5.9 \\
\hline $\begin{array}{l}\text { P3 (2o } \\
\text { Ciclo) }\end{array}$ & 0.0 & 0.0 & 26.3 & 73.7 & 77.8 & 22.2 & 0.0 & 0.0 \\
\hline
\end{tabular}

Jornadas de Formación e Innovación Docente del Profesorado | № 1 (2018)

(c) (i) $\odot$ Esta obra se distribuye con la licencia Creative Commons Reconocimiento-NoComercial-SinObraDerivada $\quad 4.0$ Internacional (CC BY-NC-ND 4.0.) 
A modo de ejemplo, se adjunta la representación en escaleras de aprendizaje correspondiente a la pregunta 1B de la Tabla 1.

\section{CUESTIONARIO \\ INICIAL}

\section{B}

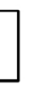

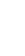

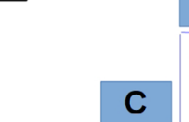

$35,3 \%$

$0.0 \%$

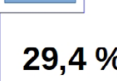

D

$35,3 \%$

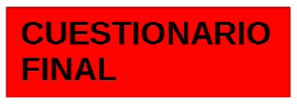

A

B

$82,4 \%$

$17,6 \%$

C

$0.0 \%$

MODELO A: Establece el orden de reacción correcto y señala que depende de si el mecanismo es simple o complejo; MODELO B: Aún no ve que la hipótesis es válida solo si el mecanismo es simple; MODELO C: Confunde el orden de reacción u opina que debe cumplirse esta dependencia independientemente del orden; MODELO D: No sabe/No contesta.

Figura 5. Esquema representativo de la evolución de los modelos de pensamiento detectados.

\section{Evaluación de los Ciclos de Mejora puestos en práctica}

En general, la experiencia docente ha sido bastante gratificante ya que se han logrado muchos de los objetivos planteados al inicio de los ciclos, y que están íntimamente relacionados con los principios didácticos que han guiado la práctica docente, como por ejemplo: (i) la implementación de un aprendizaje más colaborativo y autónomo: dos aspectos complementarios que potencian el desarrollo integral y el aprendizaje global del alumno, donde la responsabilidad personal en el desarrollo grupal es de gran importancia (Yániz Álvarez \& Villardón Gallego, 2006). Para ello se han propuesto fichas de actividades y casos prácticos a realizar en grupos en base al principio; (ii) centrar el aprendizaje en base a problemas de aplicación práctica 
empleando el método ABP (Díaz et. al., 2005). Todo ello orientado a la vida profesional; (iii) basar el aprendizaje en la práctica, lo cual supone cambiar el eje de acción del docente, proporcionando una mayor participación e implicación del alumno en su aprendizaje (Zabalza, 2010). Por último, se ha tratado en todo momento de: (iv) promover la relación teoría-práctica en el proceso de enseñanza-aprendizaje, lo cual se ha logrado trasformando el estatus quo del profesor, estableciendo puentes permanentemente entre conocimiento y acción (Álvarez-Álvarez, 2012). De hecho, en diferentes estudios (Postareff y cols., 2007; Thomas y cols. 2004; Johannes y cols., 2012) se ha comprobado que en líneas generales una formación centrada en el análisis de la práctica en el aula resulta una estrategia exitosa en el proceso de mejora docente.

En base a esta experiencia docente en el marco de la REFID existen algunos aspectos dentro de la planificación que resultan mejorables, y otros que debido a su éxito, tanto en aplicabilidad como a nivel de resultados, incorporaré en la práctica docente habitual.

En cuanto a los aspectos mejorables creo que aportar el mapa de contenidos y preguntas al alumno aporta poco, ya que su comprensión está intimamente relacionada con el trabajo realizado en su elaboración. Por ello, en la fase final de conclusiones de los ciclos de mejora debe eludirse el uso del mapa y emplear una estrategia diferente para aclarar las ideas y centrar la atención del alumno. Además de ello, se han detectado algunos contenidos de difícil comprensión, como son: la dependencia de la ecuación integrada de velocidad y la representación lineal requerida para la obtención de los parámetros cinéticos o la relación entre las etapas de los mecanismos, la estequiometría de los reactivos y la determinación de la concentración de los intermedios de reacción en base a la hipótesis del estado estacionario. Estos aspectos deberán trabajarse en cursos posteriores empleando material complementario de apoyo, u otras fichas adicionales. 
En cuanto a los aspectos que han funcionado bien y que incorporaré a la práctica docente habitual destacar: (i)

el empleo de fichas dirigidas, que ha funcionado muy bien tanto en clases de teoría como en prácticas, y ha ayudado a clarificar muchas ideas y conceptos al alumno, al tiempo que ha fomentado el trabajo en equipo y la reflexión crítica sobre los contenidos; (ii) el empleo de casos prácticos a propuesta del alumno junto con las preguntas clave basadas en problemas de la vida profesional, ha ayudado a despertar el interés del alumnado por la asignatura y a fomentar su estudio. Por todo ello, trataré en la medida de los posible incorporar también esta estrategia.

Jornadas de Formación e Innovación Docente del Profesorado | № 1 (2018) Esta obra se distribuye con la licencia Creative Commons 


\section{Referencias Bibliográficas}

Álvarez-Álvarez, C. La relación teoría-práctica en los procesos de enseñanza-aprendizaje. Educatio Siglo XXI. 2012; 30 (2): 1-20.

Díaz M, D. M; Alfaro Rocher, I. J; Apodaca Urquijo, P; Arias Blanco J M; García Jiménez E; Lobato Fraile C; Pérez Boullosa, A. (2005). Modalidades de enseñanza basadas en el desarrollo de competencias. Orientaciones para promover el cambio metodológico en el EEES. Oviedo: Ediciones Universidad de Oviedo.

Finkel, D. L. Dar clase con la boca cerrada. Publicacions de la Universitat de València. 2008; 1-292.

Johannes, C.; Fendler, J. y Seidel, T.' (2012). “Teachers' perceptions of the learning environment and their knowledge base in a training program for novice university teachers", International Journal for Academic Development, 18'(2), págs. 152-165.

Postareff, L.; Lindblom, S. y Nevgi, A. (2007a). "The effect of pedagogical training on teaching in higher education". Teaching and Teacher Education, 23, págs. 557-571.

Stephen E, D. Too much content, not enough thinking, and too little FUN!. Advances in Physiology Education. 2009; 33: 257-264.

Thomas, S.; Chie, Q. T.; Abraham, M.; Raj, S. J. y Beh, L. S. (2014). "A Qualitative Review of Literature on Peer Review of Teaching in Higher Education: An Application of the SWOT Framework (2014)". Review of Educational Research, 84 (1) págs. 112-159.

Yániz Âlvarez de Eulete, C \& Villardón Gallego, L. (2006). Planificar desde competencias para promover el aprendizaje. El reto de la sociedad del conocimiento para el profesorado universitario. Cuadernos monográficos del ICE, num. 12. Bilbao: Ediciones Deusto Digital.

Zabalza, M. A. (2010). El prácticum en la formación universitaria: estado de la cuestión. Revista de Educación, 354, 21-43. 